\title{
CARACTERIZAÇÃO DA SUPERFÍCIE DE PARTÍCULAS DE PRODUTOS LÁCTEOS DESIDRATADOS
}

\section{Characterization of the particles surface of dried dairy products}

\author{
Paulo Henrique de Melo Toledo ${ }^{1}$, João Pablo Fortes Pereira ${ }^{2}$, Ítalo Tuler Perrone ${ }^{3}$, Antônio \\ Fernandes Carvalho ${ }^{2}$, Luiz Fernando Cappa Oliveira ${ }^{1}$, Rodrigo Stephani ${ }^{1 *}$
}

\section{RESUMO}

Durante o processo de produção ou estocagem dos lácteos desidratados, os constituintes do leite podem sofrer redistribuições, resultando em uma heterogeneidade entre a superfície e seu interior. O principal objetivo deste trabalho foi realizar o mapeamento das superfícies de diferentes produtos lácteos desidratados, com o uso combinado da espectroscopia Raman e a microscopia eletrônica de varredura (MEV) acoplada à espectroscopia por energia dispersiva (EDS). Caracterizou-se as superfícies das partículas em ambos os instrumentos e realizou-se o mapeamento, dentro de populações específicas. A proposta de associar as diferentes técnicas visou, além de obter dados complementares, mostrar que é possível avançar na pesquisa dos produtos lácteos, quando se analisa as partículas sob diferentes perspectivas analíticas. Todas as análises foram realizadas a partir de produtos industrializados com lotes, datas de fabricação e validade monitoradas. A partir da análise dos perfis espectroscópicos das partículas foi possível verificar diferenças tanto do conjunto de partículas bem como das partículas alvo isoladamente. Foi possível realizar a caracterização das partículas utilizando diferentes técnicas analíticas fornecendo um conjunto de dados que amplia informações acerca das partículas dos pós estudados.

Palavras-chave: espectroscopia por energia dispersiva, mapeamento Raman, microscopia eletrônica de varredura.

1 Universidade Federal de Juiz de Fora, Departamento de Química, Rua José Lourenço Kelmer, s/n, São Pedro, 36036-900, Juiz de Fora, MG, Brasil. E-mail: rodrigostephani@gmail.com

2 Universidade Federal de Viçosa, Departamento de Tecnologia de Alimentos, Viçosa, MG, Brasil.

3 Universidade Federal de Juiz de Fora, Faculdade de Farmácia, Juiz de Fora, MG, Brasil.

* Autor para correspondência

Recebido / Received: 27/05/2019

Aprovado / Approved: 17/03/2020 


\begin{abstract}
During production or storage of dehydrated dairy products, milk constituents may undergo redistribution, resulting in heterogeneity between the surface and its interior. The main objective of this work was to map the surfaces of different dehydrated dairy products, with the combined use of Raman spectroscopy and scanning electron microscopy (SEM) coupled with energy dispersive spectroscopy (EDS). It was intended to characterize the surfaces of the particles of interest in both instruments and to perform the mapping of these, within specific populations. The proposal to combine different techniques aimed, in addition to obtaining complementary data, to show that it is possible to advance in the research of dairy products when analyzing the particles under different analytical perspectives. All analyzes were carried out from industrialized products with lots, manufacture and validity dates monitored. From the spectroscopic profiles of the particles, it was possible to verify differences when the set of particles was analyzed, as well the target particles alone. It was possible to perform the characterization of the particles using different analytical techniques providing a data set that expands information about the particles of the dairy powders analyzed.
\end{abstract}

Keywords: energy dispersive spectroscopy, Raman mapping, scanning electron microscopy.

\section{INTRODUÇÃO}

A matriz do leite é de grande complexidade, sendo constituída por aproximadamente cem mil constituintes distintos, mas a maioria deles ainda não foram identificados (SILVA, 1997). Devido à composição química e à sua micro e nanoestrutura, podemos definir o leite como um material complexo, no qual há uma emulsão de gordura e uma dispersão coloidal de proteínas em uma solução aquosa de sais, vitaminas, peptídeos e lactose (EVANS et al., 2017).

Leite e seus derivados podem ser preservados pela retirada da água por meio da concentração utilizando-se calor ou tecnologia de membrana seguida da secagem. A desidratação a partir do leite fluido confere vantagens, seja pela redução de custos de armazenamento e transporte, como no aumento do tempo de conservação do alimento (CARVALHO et al., 2016).

Os produtos lácteos desidratados são produzidos por spray dryer através da pulverização ou atomização, onde se presume que os componentes do leite estão distribuídos uniformemente pela solução. No entanto, durante o processo de secagem, esses componentes são redistribuídos, resultando em uma heterogeneidade entre a superfície e o núcleo da partícula. A distribuição superficial dos componentes depende de uma grande variedade de fatores relacionados ao processo e/ou às propriedades líquidas iniciais (KIM et al. 2009; MURRIETA-PAZOS et al., 2012). Em tecnologias mais avançadas, Zhong et al. (2017) demonstraram que a intensidade do tratamento térmico para produção do leite em pó pode afetar sua composição, microestrutura, estado de aglomeração e suas propriedades em geral, como comportamento de fluxo e característica de reidratação. Portanto, o controle de qualidade desses produtos é de grande importância.

Diferentes técnicas analíticas estão sendo utilizadas para melhorar a investigação da 
superfície dos produtos lácteos desidratados. O uso concomitante de técnicas como a microscopia eletrônica de varredura (MEV), espectroscopia de raios $\mathrm{X}$ por dispersão em energia (EDS), espectroscopia de fotoelétrons excitados por raios X (XPS), permitiram o desenvolvimento de modelos de composição de gradientes para pós integrais e desnatados aglomerados ou não (MURRIETA-PAZOS et al., 2012).

A espectroscopia Raman é adequada para análise de amostras sólidas e líquidas, fornecendo dados qualitativos e quantitativos. É um método analítico não destrutivo onde objetos estudados não requerem preparação especial e os espectros são obtidos para amostras em seu estado natural, simplificando assim as análises. A espectroscopia Raman tem sido utilizada para controle de qualidade e análises quantitativas de leite em pó, e para amostras adulteradas com soro e melamina (MAZUREK et al., 2015). A espectroscopia Raman é uma técnica versátil, rápida, determina mais de um componente ao mesmo tempo, não utilizando reagentes e não gerando subprodutos tóxicos. Além disso, as amostras não necessitam de pré-tratamento (ALMEIDA et al., 2011).

A espectroscopia de fotoelétrons excitados por raios X (XPS) fornece informações elementares (porcentagens atômicas) e químicas (porcentagens de ligação) dos primeiros 5 a $10 \mathrm{~nm}$ de profundidade da partícula de pó. A partir dos valores atômicos, as determinações das porcentagens dos principais componentes presentes na superfície dos lácteos em pó (proteína, lactose e lipídios) podem ser feitas com o auxílio de uma fórmula de matriz (MURRIETA-PAZOS et al., 2012).

Outra maneira clássica de caracterizar a superfície de lácteos em pó é o uso da microscopia eletrônica de varredura (MEV). Diferentes teores de lactose, lipídios e proteínas foram relacionados à topografia da superfície: pós túrgidos, lisos ou irregulares. No entanto, qualquer quantificação com esta técnica era impossível. A investigação da superfície dos pós a uma profundidade de 1 $\mu \mathrm{m}$ é possível com a ajuda da espectroscopia de raios $\mathrm{X}$ por dispersão em energia (EDS). Esta técnica foi realizada apenas para pós inorgânicos e farmacêuticos, mas no campo de alimentos em pó ainda não tinha sido relatado seu uso. Faltam informações sobre o gradiente de composição desde a superfície até o núcleo para pós de leite clássicos, como pós de leite integral e pós de leite desnatado variáveis (MURRIETA-PAZOS et al., 2012). A composição da superfície de pós aglomerados ainda não foi amplamente estudada merecendo assim atenção neste trabalho.

O presente trabalho realizou o mapeamento das superfícies combinando a espectroscopia Raman com a microscopia eletrônica de varredura (MEV) acoplada à energia dispersiva (EDS). A proposta de associar as diferentes técnicas visou, além de obter dados complementares, mostrar que é possível avançar na pesquisa dos produtos lácteos quando se analisam as partículas sob diferentes perspectivas analíticas. Nesse sentido, objetivou-se caracterizar as superfícies das partículas de interesse utilizando ambas as técnicas citadas acima e realizar o mapeamento destas, dentro de populações específicas, a fim de gerar informações relevantes sobre a composição das superfícies.

\section{MATERIAIS E MÉTODOS}

\section{Amostras}

As amostras utilizadas neste trabalho foram obtidas a partir de produtos industrializados com lotes, datas de fabricação e validade conhecidos, pertencentes a 4 diferentes marcas de mercado. Foram 
analisadas 3 amostras de cada produto, provenientes de diferentes lotes, sendo as análises realizadas em triplicata. Realizouse a análise de seis diferentes produtos, sem tratamento das amostras, sendo: leite em pó integral (LPI), creme de leite em pó (CR), leite em pó desnatado (LPD), soro em pó parcialmente desmineralizado (SO), concentrado proteico de leite (MPC, na sigla em inglês), concentrado proteico de soro (WPC, na sigla em inglês), sendo produtos originados a partir da desidratação do leite e do soro.

\section{Instrumentação e métodos de análise}

A morfologia, a distribuição e o tamanho das partículas das amostras dos produtos lácteos desidratados foram avaliados por microscopia eletrônica de varredura modelo Hitachi TM 3000 (Hitachi Ltd., Tokyo, Japan). A microscopia eletrônica de varredura foi utilizada para a escolha das partículas alvo para as posteriores análises. Foram utilizadas diferentes magnificações para a caracterização das amostras. A espectroscopia de raios $\mathrm{X}$ por dispersão em energia (EDS) forneceu a composição semiquantitativa dos elementos químicos presentes nas partículas alvo escolhidas.

Para a espectroscopia Raman foi utilizado o espectrômetro FT-Raman da marca Bruker, modelo RFS/100, utilizado como radiação excitante a linha laser $\lambda_{0}=1064 \mathrm{~nm}$ (laser de $\mathrm{Nd} / \mathrm{YAG}$ ), com potência de $85 \mathrm{~mW}$ e 512 varreduras. Esta análise forneceu informações sobre a macrocomposição dos principais componentes das amostras como lipídios, proteínas e lactose.

Para a análise das partículas alvo escolhidas através do MEV e sua identificação foi utilizado o espectrômetro dispersivo Raman com microscopia óptica Bruker, modelo SENTERRA. Os dados espectrais foram coletados utilizando radiação excitante com linha laser $\lambda_{0}=632,8 \mathrm{~nm}$. A potência laser utilizada foi de $10 \mathrm{~mW}$. A objetiva de magnificação foi de $50 \mathrm{X}$, gerando um foco de $4 \mu \mathrm{m}$ (DENECKERE, 2012). O tempo de integração de 25 segundos e 4 condições. Esses foram os parâmetros utilizados para cada análise. Os dados fornecidos através da espectroscopia Raman no equipamento SENTERRA forneceu informações complementares sobre a composição das populações alvo, através das bandas espectrais características de alguns componentes (lipídeos, proteínas e lactose), que são os principais componentes encontrados nas amostras, e suas bandas características são descritas na literatura.

As análises da composição das partículas alvo presentes nas amostras, a partir de diferentes técnicas, serviram como parâmetros de comparação da composição entre as mesmas e o seu conjunto, nas diferentes amostras.

\section{RESULTADOS E DISCUSSÃO}

Nas Figuras 1 e 2 são apresentadas as imagens das partículas alvo provenientes de amostras de lácteos desidratados, sendo em A, obtidas por microscopia eletrônica de varredura e em B, obtidas por microscopia óptica, com objetiva de magnificação de 10 vezes (10X).

A partir da observação das imagens em diferentes perspectivas analíticas foi possível identificar as partículas alvo das amostras. A identificação das partículas alvo na microscopia óptica só foi possível através da análise das partículas vizinhas a partícula alvo e comparação destas com as imagens obtidas na microscopia eletrônica de varredura. A obtenção das imagens na microscopia óptica foi importante, pois possibilitou a posterior obtenção do perfil espectral das partículas alvo, trazendo uma maior especificidade do resultado, possibilitando assim a verificação 

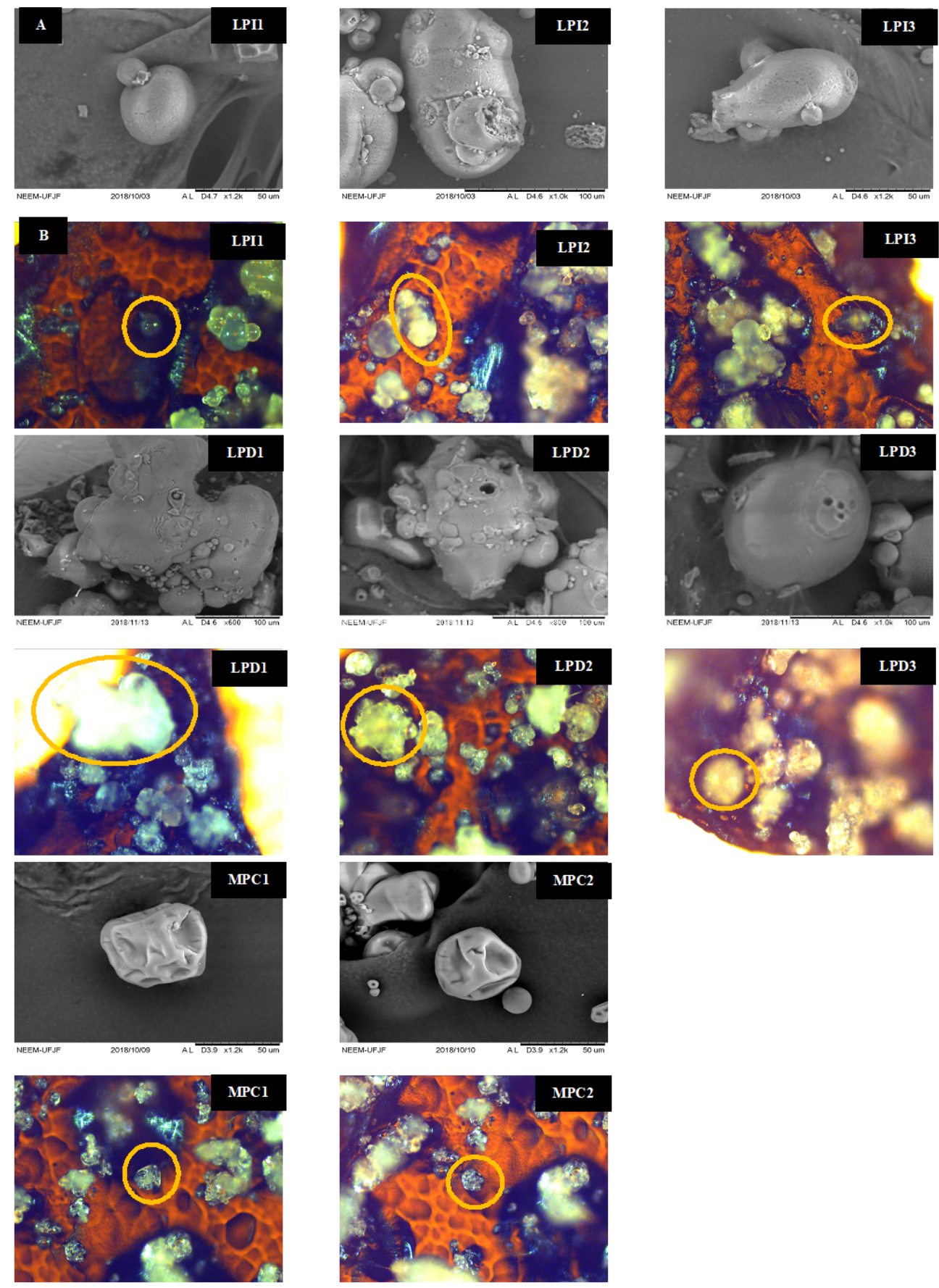

Figura 1 - Partículas alvo dos produtos lácteos desidratados identificadas no MEV (A) e na microscopia óptica (B): LPI (leite em pó integral); LPD (leite em pó desnatado); e MPC (concentrado proteico de leite) 

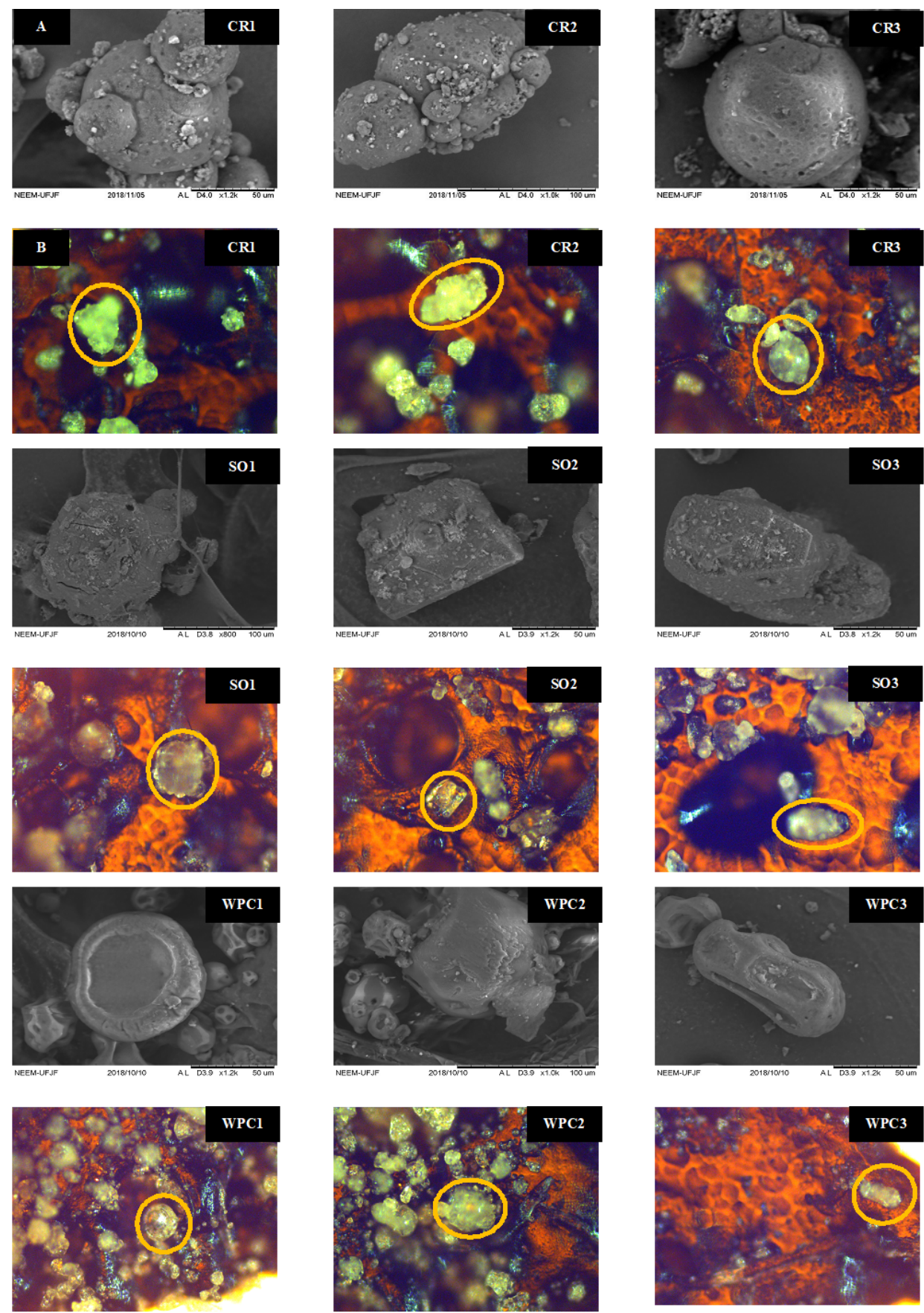

Figura 2 - Partículas alvo dos produtos lácteos desidratados identificadas no MEV (A) e na microscopia óptica (B): CR (creme de leite em pó); SO (soro de leite em pó parcialmente desmineralizado); e WPC (concentrado proteico de soro) 
da heterogeneidade das partículas. Isso viabilizou uma diferenciação de análises em que se observou o perfil espectral do conjunto de partículas.

As partículas apresentaram morfologias, tamanhos e aspectos de superfícies diferentes. A não homogeneidade morfológica demonstrada para uma mesma amostra é resultante do processo de produção e estocagem. Características inerentes ao processo e intrínsecas à composição e interações químicas dos produtos antes da secagem são responsáveis por tal modelagem. As partículas de leite em pó integral e desnatado apresentaram-se individualizadas, sem a presença de partículas adjacentes diretamente ligadas, indicando tratar-se de produtos obtidos sem aglomeração significativa durante a secagem, característico de sistemas de secagem de único estágio.

As imagens do MPC na Figura 1 apresentaram morfologia destacada, devido às invaginações apresentadas, o que pode ser resultante da desidratação da partícula rica em caseína. A caseína é uma micela altamente solvatada, antes do processo de secagem.

Na Figura 2 observa-se que as partículas de creme se apresentaram aglomeradas, indicando que podem ter sido produzidas em equipamento de secagem de múltiplos estágios ou que o produto estaria realizando interações entre os lipídeos de superfície. As imagens de WPC mostram que as partículas não se encontravam túrgidas, apresentando regiões murchas. Esta morfologia é esperada de produtos como o WPC que perdem grande parte da umidade dentro do spray dryer, uma vez que a quantidade de água que é possível evaporar a vácuo é limitada.

Nas imagens do SO observou-se a forma de "Tomahawk", característica do cristal de lactose. Os soros em pós apresentam aproximadamente $75 \%$ de lactose em sua composição, dos quais $70 \%$ encontram-se normalmente cristalizadas (SIMEÃO et al., 2017).
Na Figura 3 são apresentados os espectros Raman das amostras dos produtos lácteos desidratados.

Analisando os espectros das diferentes amostras, foram observadas diferenças entre o perfil das partículas de uma mesma amostra. $\mathrm{O}$ mesmo comportamento foi percebido quando comparados os espectros de cada uma das partículas (em vermelho) e o espectro obtido do conjunto de partículas da amostra (em preto). A qualidade dos espectros obtidos no SENTERRA, utilizando a radiação excitante $\lambda_{0}=632,8 \mathrm{~nm}$, é dependente da qualidade da focalização da partícula alvo. Partículas planares são mais bem focalizadas, por conseguinte possibilitam a obtenção de espectros de melhor qualidade. Wieboldt (2010) ao detalhar os parâmetros para a obtenção de um bom espectro Raman, destaca a importância da focalização adequada e os limites do material a ser analisado.

As amostras estudadas, em sua maioria, apresentaram formas não planares, sendo esféricas ou ovais, o que gerou ruído e fluorescência nos espectros obtidos. A fluorescência pode ser também proveniente da composição química das partículas analisadas. Interferência na linha de base por fluorescência é um problema comum em amostras biológicas (YANG et al., 2017). Como exemplo, o creme de leite em pó apresentou menor fluorescência, provavelmente devido a sua diferença composicional. Isto permitiu que os espectros obtidos nas partículas alvo tivessem maior similaridade com o perfil espectroscópico do conjunto de partículas obtido no FT-Raman.

As técnicas empregadas visaram a caracterização dos diferentes produtos lácteos desidratados, contudo a comparação entre os espectros obtidos dos pós não foi possível, uma vez que o histórico do processamento industrial não era conhecido. Trabalhos futuros podem determinar a relação entre os resultados espectroscópicos 
dos diferentes produtos a partir de pós obtidos em escala piloto com parâmetros de processamento padronizados e conhecidos. A descrição e controle rigoroso das etapas do processamento configuram o pré-requisito para uma abordagem científica robusta dos diferentes espectros obtidos, o que não foi o objetivo deste trabalho.

Na Tabela 1 são analisadas as tentativas de atribuição das bandas espectrais destacadas obtidas das diferentes amostras estudadas.
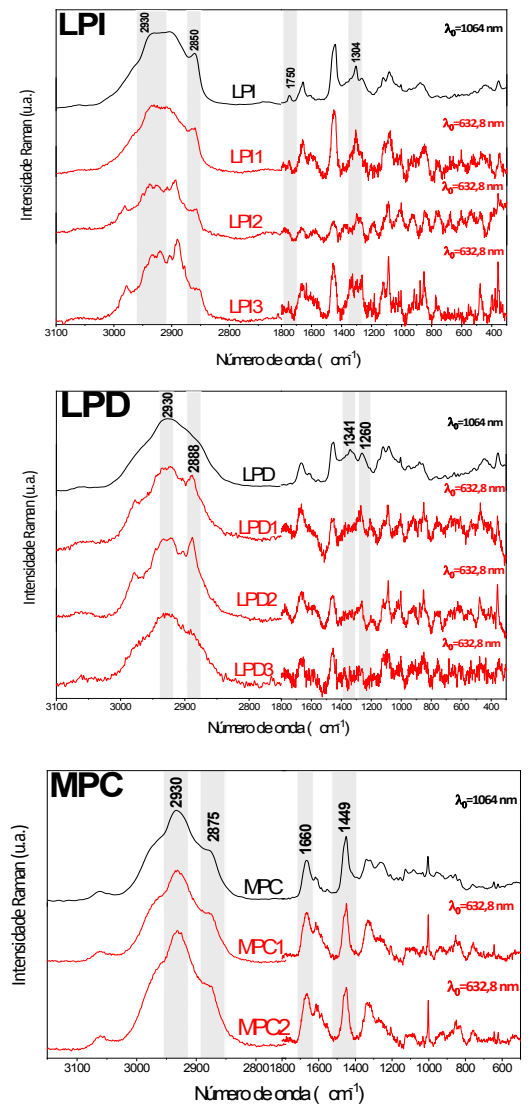

A classificação em leite integral ou desnatado é baseada na quantidade gordura presente. Nos espectros Raman, os modos vibracionais característicos podem ser encontrados comparado espectro dessas amostras. Desta forma, observou-se uma banda larga em $2930 \mathrm{~cm}^{-1}$ para o LPI a qual foi mais definida no LPD. Essa banda pode ser atribuída ao modo vibracional de estiramento assimétrico do grupo $\mathrm{CH}_{2}$, podendo ser relacionada a proteínas e carboidratos
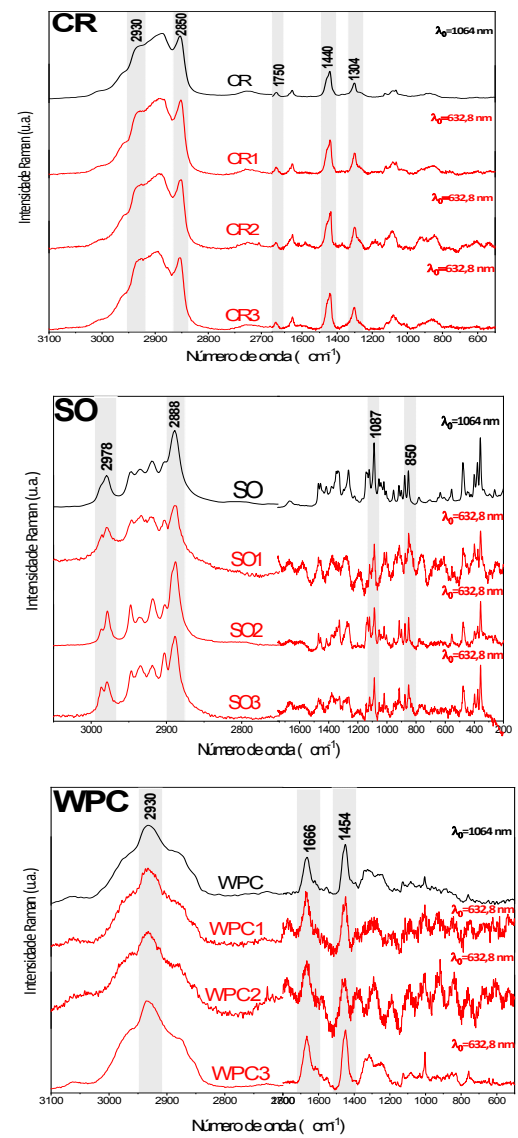

Figura 3 - Espectros Raman FT (linha preta) e Senterra (linha vermelha) dos produtos lácteos desidratados: leite em pó integral (LPI); creme de leite em pó (CR); leite em pó desnatado (LPD); soro em pó parcialmente desmineralizado (SO); concentrado proteico de leite (MPC); concentrado proteico de soro (WPC) 
(ALMEIDA et al., 2011). O mesmo raciocínio pode ser realizado ao analisar produtos com baixo teor (SO, WPC, MPC) e elevado teor de lipídio (CR).

A região de $2000 \mathrm{~cm}^{-1}$ a $400 \mathrm{~cm}^{-1}$ apresentou grande quantidade de bandas sendo rica em informação estrutural. Uma banda de fraca intensidade foi percebida na região de $1750 \mathrm{~cm}^{-1}$ das amostras de LPI e CR e não percebida nas demais amostras que são pobres em gordura, indicando que seja procedente de modo vibracional de lipídios. Esta banda é atribuída ao modo vibracional de estiramento do grupo $\mathrm{C}=\mathrm{O}$ dos ácidos graxos presentes no leite (ZHOU et al., 2006).

No SO e WPC destacaram-se bandas referentes à alta concentração de lactose cristalina que se divide em duas regiões. A primeira região compreendida entre $2980 \mathrm{~cm}^{-1}$ e $2880 \mathrm{~cm}^{-1}$ foi atribuída a modos vibracionais relacionados ao grupamento $\mathrm{CH}$ presente em estruturas aromáticas e alifáticas. A segunda região localizada entre $1120 \mathrm{~cm}^{-1}$ e $850 \mathrm{~cm}^{-1}$, possui diversos modos vibracionais destacando os modos acoplados $v(\mathrm{C}-\mathrm{O})+v(\mathrm{C}-\mathrm{C})+$ $\delta(\mathrm{C}-\mathrm{O}-\mathrm{H})$ e $v(\mathrm{C}-\mathrm{O}-\mathrm{C})+\delta(\mathrm{C}-\mathrm{O}-\mathrm{H})+v(\mathrm{C}-\mathrm{O})$ (STEPHANI et al.; 2017). Assim, o uso da técnica de espectroscopia Raman permitiu a caracterização dos materiais analisados.

Na Tabela 2 são apresentados os resultados da composição da superfície das partículas alvo, obtidas por meio da espectroscopia de raios $\mathrm{X}$ por dispersão em energia (EDS).

Tabela 1 - Tentativa de atribuição de bandas Raman

\begin{tabular}{cc}
\hline Número de onda $\left(\mathrm{cm}^{-1}\right)$ & Tentativa de atribuição \\
\hline 2978 & $v(\mathrm{C}-\mathrm{H})$ \\
2930 & $v_{\text {ass }}\left(\mathrm{CH}_{2}\right)$ \\
2888 & $v(\mathrm{C}-\mathrm{H})$ \\
2880 & $v_{\text {ass }}\left(\mathrm{CH}_{3}\right)$ \\
2875 & $v_{\text {ass }}\left(\mathrm{CH}_{2}\right)+v_{\mathrm{s}}\left(\mathrm{CH}_{3}\right)+v_{\mathrm{s}}\left(\mathrm{CH}_{2}\right)$ \\
2850 & $v_{\mathrm{s}}\left(\mathrm{CH}_{2}\right)$ \\
1750 & $v_{\text {ass }}(\mathrm{C}=\mathrm{O})_{\text {ester }}$ \\
1660 & $\mathrm{v}(\mathrm{C}=\mathrm{O}) \mathrm{Amida} \mathrm{I}^{\mathrm{v}}(\mathrm{C}=\mathrm{C})$ \\
1454 & $\delta\left(\mathrm{CH}_{2}\right)$ \\
1440 & $\delta\left(\mathrm{CH}_{2}\right)$ \\
1341 & $v(\mathrm{C}-\mathrm{H})+\mathrm{v}(\mathrm{C}-\mathrm{O})$ \\
1304 & ${ }_{\tau}\left(\mathrm{CH}_{2}\right)$ \\
1280 & ${ }_{\tau}\left(\mathrm{CH}_{2}\right)$ \\
1087 & $v(\mathrm{C}-\mathrm{O})+\mathrm{v}(\mathrm{C}-\mathrm{C}-\mathrm{C})+\delta(\mathrm{C}-\mathrm{O}-\mathrm{H})$ \\
850 & $(\mathrm{C}-\mathrm{O}-\mathrm{H})+\mathrm{v}(\mathrm{C}-\mathrm{O})$ \\
\hline &
\end{tabular}


A análise por EDS é semiquantitativa, permitindo a obtenção de bons indícios acerca da composição das superfícies das partículas. Como esperado, por se tratar de compostos alimentícios, houve um predomínio dos elementos carbono e oxigênio, destacando também a presença de minerais como cálcio, fósforo e potássio. Destaca-se a presença de elementos atípicos à composição desses alimentos $\mathrm{c}^{`}$ mo alumínio e silício. $\mathrm{O}$ alumínio é provavelmente, proveniente da manipulação industrial para a fabricação e envase dos produtos estudados. Stahl et al. (2017) encontraram alumínio em diversos gêneros alimentícios vegetais e animais, destacando que, embora possa compor o alimento naturalmente devido a ingestão de água e vegetação contendo alumínio, a

Tabela 2 - Dados de microanálise obtidos em EDS dos produtos lácteos desidratados (percentual atômico de elementos*)

\begin{tabular}{|c|c|c|c|c|c|c|c|c|c|c|}
\hline & C & $\mathbf{O}$ & Al & $\mathrm{Ca}$ & $\mathbf{P}$ & $\mathbf{K}$ & Cl & $\mathbf{S}$ & $\mathbf{N a}$ & $\mathbf{S i}$ \\
\hline LPI1 & 66,38 & 28,48 & 3,10 & 0,87 & 0,68 & 0,48 & - & - & - & - \\
\hline LPI2 & 56,21 & 32,24 & 5,22 & 2,49 & 1,49 & 2,03 & - & 0,33 & - & - \\
\hline LPI3 & 59,07 & 31,52 & 4,36 & 1,92 & 1,78 & 0,94 & - & 0,42 & - & - \\
\hline LPD1 & 24,14 & 46,86 & 20,45 & 2,49 & 1,44 & 2,51 & 1,49 & - & 0,62 & - \\
\hline LPD2 & 44,08 & 43,75 & 6,37 & 1,58 & 0,73 & 1,97 & 0,85 & 0,15 & 0,51 & - \\
\hline LPD3 & 56,91 & 36,65 & 4,77 & 0,35 & 0,27 & 0,47 & 0,27 & - & 0,32 & - \\
\hline MPC1 & 58,01 & 32,98 & 4,41 & 2,13 & 1,32 & 0,58 & - & 0,56 & - & - \\
\hline MPC2 & 59,15 & 34,98 & 2,97 & 1,68 & 1,24 & - & - & - & - & - \\
\hline CR1 & 68,15 & 26,99 & 3,72 & - & - & - & - & - & - & 1,14 \\
\hline CR2 & 69,35 & 25,53 & 3,53 & - & - & 0,65 & - & - & - & 0,95 \\
\hline CR3 & 65,75 & 31,28 & 2,45 & - & - & 0,52 & - & - & - & - \\
\hline SO1 & 47,04 & 42,24 & 3,42 & 1,00 & 1,35 & 3,20 & 0,81 & - & 0,94 & - \\
\hline SO2 & 52,18 & 40,53 & 2,64 & 0,59 & 1,05 & 1,60 & 0,53 & - & 0,87 & - \\
\hline SO3 & 54,20 & 38,74 & 2,64 & - & 1,37 & 2,22 & - & - & 0,83 & - \\
\hline WPC1 & 50,74 & 40,99 & 5,16 & 0,69 & 0,34 & 0,76 & - & 1,33 & - & - \\
\hline WPC2 & 59,81 & 32,46 & 5,73 & 0,63 & 0,27 & 0,26 & - & 0,84 & - & - \\
\hline WPC3 & 53,88 & 32,04 & 9,99 & 1,79 & 0,20 & 0,42 & - & 1,25 & 0,43 & - \\
\hline
\end{tabular}

* Sendo $\mathrm{C}=$ carbono $\mathrm{O}=$ oxigênio $; \mathrm{Al}=$ alumínio $\mathrm{P}=$ fósforo $\mathrm{K}=$ potássio $\mathrm{Cl}$ = cloro; $\mathrm{S}=$ enxofre; $\mathrm{Na}=$ sódio; $\mathrm{Si}=$ silício. 
maior parte do metal é oriunda do processo de industrialização do alimento. O silício foi encontrado na amostra de creme de leite em pó, devido à aspersão de dióxido de silício posterior a secagem devido a sua função antiaglutinante durante a estocagem.

\section{CONCLUSÃO}

A microscopia eletrônica de varredura forneceu informações importantes sobre a morfologia das partículas e foi determinante para a escolha de partículas alvo utilizadas para a determinação semiquantitativa dos elementos químicos da superfície e para a obtenção de seus perfis espectroscópicos. A partir da análise dos perfis espectroscópicos das partículas obtidas por diferentes equipamentos, foi possível verificar diferenças quando foi analisado o conjunto de partículas através do espectrômetro FT Raman, e as partículas alvo isoladamente através do espectrômetro dispersivo SENTERRA. Foi possível realizar a caracterização das partículas utilizando diferentes técnicas analíticas fornecendo um conjunto de dados que amplia informações acerca das partículas dos pós estudados.

O presente trabalho apresentou uma técnica que possibilita estudos de caracterização de partículas específicas de diferentes materiais abrindo opções de pesquisa na área. Isto é importante para o melhor entendimento e caracterização do comportamento de produtos lácteos em pó. Os métodos empregados mostraram-se eficientes na caracterização das superfícies das partículas em pó analisadas.

Em estudo futuro sugere-se realizar tanto a produção dos produtos em condições controladas e conhecidas, bem como a determinação da composição dos produtos visando determinar o efeito do processamento e da composição nas características das superfícies dos pós.

\section{REFERÊNCIAS}

ALMEIDA, M. R.; STEPHANI, R.; OLIVEIRA, L. F. C. Fourier-transform Raman analysis of milk powder: A potential method for rapid quality screening. Journal of Raman Spectroscopy, v. 42, n. 7, p. 1548-1552, 2011.

CARVAlHO, A. F.; MARTINS, E.; RODRIGUES, R. C.; SCHUCK, P.; PERRONE, I. T. Tecnologia de lácteos concentrados e desidratados: O estado da arte da microbiologia de processo. Indústria de Laticínios, n. 121, p. 71-73, 2016.

DENECKERE, A. Development, optimization and application of Raman spectroscopic and $\mathrm{X}$-ray fluorescence spectroscopic methodology in the field of cultural heritage. 2012. Dissertation (Doctor Ph.D. in Sciences, Chemistry) - Faculty of Sciences, Ghent University, Ghent, 2012.

EVANS, A. A.; CHEUNG, E.; NYBERG, K. D.; ROWAT, A. C. Wrinkling of milk skin is mediated by evaporation. Soft Matter, v. 13, p. 1.056-1.062, 2017.

KIM, E. H. J.; CHEN, X. D.; PEARCE, D. Surface composition of industrial spraydried milk powders. Effects of spray drying conditions on the surface composition. Journal of Food Engineering, v. 94, p. 169-181, 2009.

MAZUREK, S.; SZOSTAK, R.; CZAJA, T.; ZACHWIEJA, A. Analysis of milk by FT-Raman spectroscopy. Talanta, v. 138, p. 285-289, 2015.

MURRIETA-PAZOS, I.; GAIANI, C.; GALET, L.; SCHER, J. Composition gradient from surface to core in dairy powders: Agglomeration effect. Food Hydrocolloids, v. 26, n.1, p. 149-158, 2012. 
SILVA, P. H. F. Leite: aspectos de composição e propriedades. Química Nova na Escola, v. 6, p.3-5, 1997.

SIMEÃO, M; SILVA, C. R.; STEPHANI, R.; OLIVEIRA, L. F. C.; SCHUCK, P.; CARVALHO, A. F.; PERRONE, I T. Lactose crystallisation in concentrated whey: The influence of vat type. International Journal of Dairy Technology, v. 71, n. 2, p. 478-483, 2018.

STAHL, T.; FALK, S.; ROHRBECK, A.; GEORGII, S.; HERZOG, C.; WIEGAND, A.; HOTZ, S.; BOSCHEK, B., ZORN, H.; BRUNN, H. Migration of aluminum from food contact materials to food - a health risk for consumers? Part I of III: Exposure to aluminum, release of aluminum, tolerable weekly intake (TWI), toxicological effects of aluminum, study design, and methods. Environmental Sciences Europe, v. 29, n. 19, p. 1-8, 2017.

STEPHANI, R.; OLIVEIRA, K. S.; ALMEIDA, C. E. R.; PERRONE, I. T.; CARVALHO, A. F.; OLIVEIRA, L. F. C.; ALMEIDA, M. R. Raman spectroscopy as a tool to identify modification of whey protein concentrate (WPC) during shelf life. Food Packaging and Shelf Life, v. 11, p. 1-9, 2017.

WIEBOLDT, D. Understanding Raman spectrometer parameters. Spectroscopy, special issue, 2010. Disponível em: http://www.spectroscopyonline.com/ understanding-raman-spectrometerparameters $? \mathrm{id}=\&$ pageID $=1 \& \mathrm{sk}=\&$ date $=$

YANG, S.; AKKUS, O.; CREASEY, D. 1064nm Raman: The right choice for biological samples? Spectroscopy, v. 32, n.6, p. 46-54, 2017.

ZHONG, Y.; WU, Y.; ZHENG, Y.; ZHU, H.; LIU, Z.; JIAO, S. Assessment of radio frequency heating on composition, microstructure, flowability and rehydration characteristics of milk powder. Food Science and Technology, v. 37, n. 4, p. 544-551, 2017.

ZHOU, G.; SUN, S. Q.; YU, L.; XU, C. H.; NODA, I.; ZHANG, X. R. Sequential changes of main components in different kinds of milk powders using two-dimensional infrared correlation analisys. Journal of Molecular Structure, v. 799, n. 6, p. 77-84, 2006. 